\title{
Explaining the Praesepe blue straggler HD 73666 (Research Note)
}

\author{
L. Fossati ${ }^{1,2}$, S. Mochnacki ${ }^{3}$, J. Landstreet ${ }^{4}$, and W. Weiss ${ }^{1}$ \\ 1 Institut für Astronomie, Universität Wien, Türkenschanzstrasse 17, 1180 Wien, Austria \\ e-mail: weiss@astro.univie.ac.at \\ 2 Department of Physics and Astronomy, The Open University, Milton Keynes, MK7 6AA, UK \\ e-mail: 1.fossati@open.ac.uk \\ 3 Department of Astronomy \& Astrophysics, University of Toronto, 50 St. George St, Rm.101, Toronto, ON, M5S 3H4 Canada \\ e-mail: stefan@astro.utoronto.ca \\ 4 Department of Physics \& Astronomy, University of Western Ontario, London, ON, N6A 3K7 Canada \\ e-mail: jlandstr@astro.uwo.ca
}

Received 10 December 2008 / Accepted 5 November 2009

\begin{abstract}
Aims. The blue straggler phenomenon is not yet well explained by current theory, although evolutionary models of star clusters require a good knowledge of it. We attempt to develop a formation scenario for HD 73666, a blue straggler member of the Praesepe cluster. Methods. We compile the known physical properties of HD 73666 found in the literature, focusing in particular on possible binarity and the abundance pattern.

Results. HD 73666 appears to be slowly rotating, have no detectable magnetic field, and have normal abundances, thereby excluding close binary evolution and mass transfer processes. There is no evidence of a hot radiation source.

Conclusions. With the use of theoretical results on blue straggler formation present in literature, we are able to conclude that HD 73666 was probably formed by physical collision involving at least one binary system, between 5 and 350 Myr (50 Myr if the star is an intrinsic slow rotator) ago.
\end{abstract}

Key words. blue stragglers - open clusters and associations: general - stars: formation - stars: individual: HD 73666

\section{Introduction}

Blue stragglers are stars that lie on an extension of the main sequence and are bluer and brighter than the main-sequence turn-off stars. These objects are found in star clusters, dwarf galaxies, and in the field. The existence of blue stragglers can probably be explained only by an interaction between two or more stars, and so to understand this phenomenon we study the interaction of stars in stellar systems (see Leonard 1989; Bailyn \& Pinsonneault 1995; Sandquist et al. 1997; Sills et al. 1997; Chen \& Han 2004; Ahumada \& Lapasset 2007).

Ahumada \& Lapasset (2007) listed the most frequently cited explainations of the formation of blue stragglers. They could be (1) horizontal-branch stars that appear above the main sequence turn-off point; (2) stars of a second or third generation; (3) stars that have extended their main-sequence life because of some internal mixing, which would generate a chemically peculiar blue straggler; (4) stars formed by the collision of two single stars; (5) the result of mass transfer in a close binary system; (6) produced by the merger of the components of a binary system; or (7) the result of a collision between two or more binary systems. Of those seven theories they, considered the last four as the most likely channels of formation.

Comprehensive catalogues of blue stragglers in open and globular clusters have been published. Ahumada \& Lapasset (1995) created the first consistent catalogue of blue stragglers in open clusters, which was then expanded by de Marchi et al. (2006) and finally superseded by Ahumada \& Lapasset (2007). These catalogues make it possible to analyse blue stragglers on a solid statistical base, leading, for example, to the conclusion that the number of blue stragglers increases with cluster age (see Fig. 5 by Ahumada \& Lapasset 2007). In the light of this result, evolutionary models of open clusters need to consider this poorly understood phenomenon. For this reason, it is important to find clues that allow us to identify the most probable blue straggler formation channels.

The A1V star HD 73666 (40 Cnc, $V=6.61$ ) is an extreme blue straggler (Ahumada \& Lapasset 2007) in the nearby, wellstudied cluster Praesepe (NGC 2632). This paper aims to show that it is a particularly important example that provides an excellent test case for theories of blue straggler formation. This paper can also be seen as the continuation of Conti et al. (1974) in the light of more than thirty years of new astronomical knowledge and instrumental development.

In Sect. 2, we discuss the membership of HD 73666 in the Praesepe cluster. Fundamental parameters and other physical properties are given in Sect. 3, where we show that the star is a blue straggler. In Sect. 5, we describe the possible formation scenario. Our conclusions are gathered in Sect. 6.

\section{Cluster membership}

The determination of cluster membership is the first step in demonstrating that a star is a blue straggler. For this purpose, the Praesepe cluster is a perfect object to study because it has proper motions and a mean radial velocity that are distinct from those of the field stars in its vicinity. This peculiarity can be seen in 


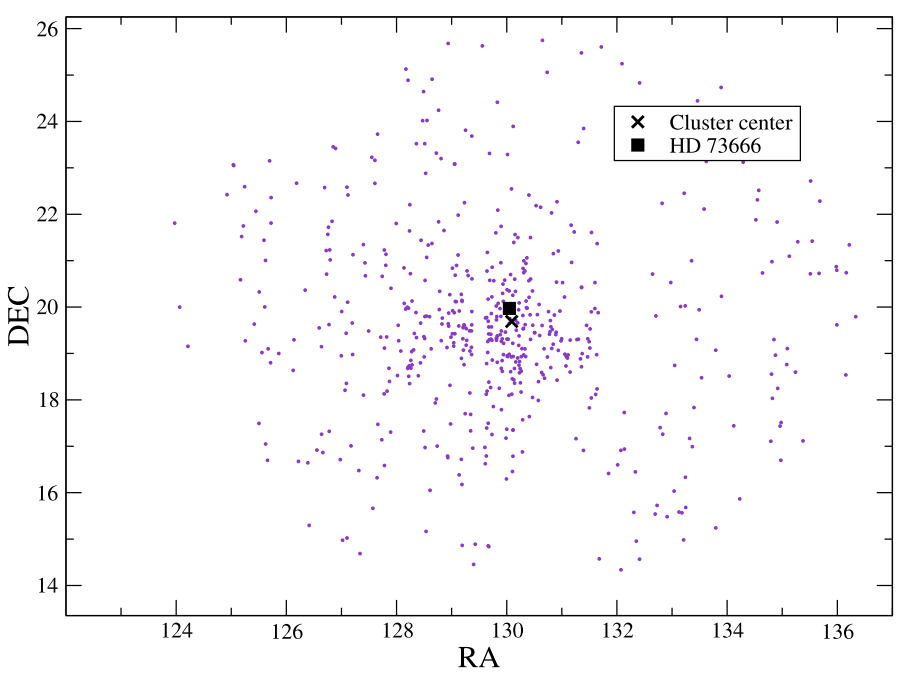

Fig. 1. Location of HD 73666 relative to stars with a membership probability higher than $40 \%$ as tabulated in Adams et al. (2002). The center of the cluster is also shown.

the atlas of the Praesepe cluster published by Kharchenko et al. (2005), who show the proper motions of the cluster members, concentrated around the mean $(-35.90,-12.88)$ mas/yr, compared with the motions of the field stars lying in the same region of $(-8.68,-1.37) \mathrm{mas} / \mathrm{yr}$ with a dispersion, for the cluster stars, of $\sigma_{\mu_{\mathrm{AF}}}=13.58 \pm 3.71 \mathrm{mas} / \mathrm{yr}$ (Kharchenko et al. 2004).

Major studies of cluster membership were published by Wang et al. (1995), Robichon et al. (1999), Baumgardt et al. (2000), Kharchenko et al. (2004), and Dias et al. (2006). HD 73666 was assumed by Robichon et al. (1999) and Baumgardt et al. (2000) to be a cluster member, and used to derive the mean cluster astrometric parameters and mean radial velocity. Kharchenko et al. (2004) also considered HD 73666 to be a cluster member having a kinematic membership probability of 0.3175 , a photometric membership probability of 1.0000 , and a positional probability of 1 (Kharchenko et al. considers every star with a kinematic and photometric membership probability higher than 0.14 as a probable member of the cluster).

Table 1 shows a comparison between the cluster mean values of parallax, proper motion and radial velocity for Praesepe and those of HD 73666. The measurements of both $\mu_{\alpha} \cos (\delta)$ and the radial velocity are in agreement within one $\sigma$, but $\mu_{\delta}$ for the cluster and the star differ by $2.7 \sigma$. If one considers the cluster members listed by Robichon et al. (1999) and selects from the re-reduction of Hipparcos data by van Leeuwen (2007) 12 proper motions with measurement uncertainties comparable to those of HD 73666, it is found that the dispersion of $\mu_{\delta}$ is approximately $1.1 \mathrm{mas} / \mathrm{yr}$, a value very similar to the difference between the $\mu_{\delta}$ cluster mean of both Praesepe and HD 73666. Thus, the apparent discrepancy between the cluster and star values of $\mu_{\delta}$ is typical of all the most precise $\mu_{\delta}$ values for the cluster. The origin of this effect is unclear to us, but our conclusion is that the agreement between cluster and stellar mean values is as satisfactory for HD 73666 as it is for most of the most precisely measured stars in the cluster, and is not a cause for concern for this specific star.

Figure 1 shows the projected location of HD 73666 relative to other cluster members, which is close to the center of the cluster. In conclusion, there is little reason to doubt that HD 73666 is a member of Praesepe.

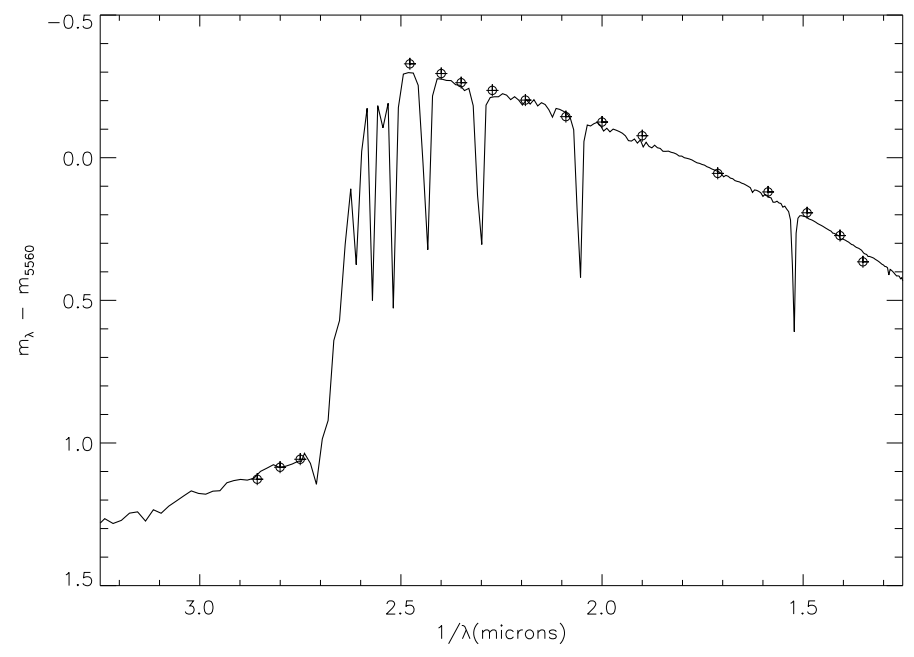

Fig. 2. Comparison between spectrophotometry from Clampitt \& Burstein (1997) and theoretical fluxes normalised to $5560 \AA$ with the fundamental parameters of HD 73666.

\section{Fundamental parameters and color-magnitude diagram}

From high resolution spectroscopy, Fossati et al. (2007) derived the $T_{\text {eff }}$ and $\log g$ of HD 73666. They obtained $T_{\text {eff }}=$ $9380 \pm 200 \mathrm{~K}$ and $\log g=3.78 \pm 0.2$. These parameters are also confirmed by spectrophotometry taken from Clampitt \& Burstein (1997). We calculated theoretical stellar flux using the model atmosphere code ATLAS9 (Kurucz 1979) with these values of $T_{\text {eff }}$ and $\log g$ and then normalised the fluxes to $5560 \AA$. The comparison between the spectrophotometry and the normalised fluxes is shown in Fig. 2.

Figure 3 shows the color-magnitude (CM) diagram of the cluster compiled using the photometry taken from Johnson (1952). In the plot, we also display two isochrones from Girardi et al. (2002) of metallicity $Z=0.024 \pm 0.002$ dex taken from Chen et al. (2003) but with different ages. The full line represents the isochrone corresponding to the cluster age of $\log t=$ $8.85 \pm 0.15$ dex (González-García et al. 2006), while the dashed line represents the isochrone that fits most accurately the position of HD 73666 in the HR diagram, corresponding to an age of $\log t=8.55$. In Fig. 3, we adopted a distance modulus of $6.30 \mathrm{mag}$ (van Leeuwen 2007) and a reddening of $0.009 \mathrm{mag}$ (Mermilliod \& Paunzen 2003; van den Bergh 2006).

The CM diagram shows clearly the blue straggler property of HD 73666. According to Fossati et al. (2008), HD 73666 appears to be about $1200 \mathrm{~K}$ hotter than the hottest main-sequence star member of the cluster. The mass of HD 73666 derived by Fossati et al. (2008), from the Hertzsprung-Russell (HR) diagram, is $2.46 \pm 0.12 M_{\odot}$. This value is about $0.4 M_{\odot}$ higher than the mass of the other most massive (of mass higher or equal to $2 M_{\odot}$ ) main-sequence cluster stars. Following the results of Fossati et al. (2008), the four most massive main sequence stars (excluding HD 73666) are: HD $73618\left(2.16 \pm 0.22 M_{\odot}\right)$, HD 72846 $\left(2.09 \pm 0.15 M_{\odot}\right)$, HD $73711\left(2.08 \pm 0.10 M_{\odot}\right)$, and HD 73709 $\left(2.00 \pm 0.14 M_{\odot}\right)$. From $T_{\text {eff }}$ and $\log L / L_{\odot}$, we derive a radius of $R / R_{\odot}=2.72 \pm 0.12$.

One possible explanation of the existence of blue stragglers is that the star is a horizontal branch star of the same temperature and luminosity as main-sequence stars. This explanation does not seem probable: the horizontal branch is bluer in a lowmetallicity environment (see e.g, Sandage \& Wallerstein 1960) 
Table 1. Proper motion and radial velocity of the Praesepe cluster and HD 73666.

\begin{tabular}{ccccccccccccc}
\hline \hline & RA & Dec & $\begin{array}{c}\pi \\
{[\mathrm{mas}]}\end{array}$ & $\begin{array}{c}\sigma_{\pi} \\
{[\mathrm{mas}]}\end{array}$ & $\begin{array}{c}\mu_{\alpha} \cos (\delta) \\
{[\mathrm{mas} / \mathrm{yr}]}\end{array}$ & $\begin{array}{c}\sigma_{\mu_{\alpha} \cos (\delta)}[\mathrm{mas} / \mathrm{yr}] \\
{[\mathrm{mas} / \mathrm{yr}]}\end{array}$ & $\begin{array}{c}\mu_{\delta} \\
{[\mathrm{mas} / \mathrm{yr}]}\end{array}$ & $\begin{array}{c}v_{r} \\
{\left[\mathrm{~km} \mathrm{~s}^{-1}\right]}\end{array}$ & $\begin{array}{c}\sigma_{v_{\mathrm{r}}} \\
{\left[\mathrm{km} \mathrm{s}^{-1}\right]}\end{array}$ & $\begin{array}{c}T_{\text {eff }} \\
{[\mathrm{K}]}\end{array}$ & $\begin{array}{c}\log L / L_{\odot} \\
{[\mathrm{dex}]}\end{array}$ \\
\hline Praesepe & 0840.4 & +1941 & 5.49 & 0.19 & -35.68 & 0.30 & -12.72 & 0.25 & 34.5 & 0.0 & $(\ldots)$ & $(\ldots)$ \\
HD 73666 & 0840.2 & +1958 & 5.53 & 0.50 & -35.52 & 0.62 & -13.97 & 0.39 & 34.1 & 0.4 & 9380 & 1.71 \\
\hline
\end{tabular}

Notes. $v_{r}, \sigma_{v_{\mathrm{r}}}$, and $T_{\text {eff }}$ of HD 73666 are taken from Fossati et al. (2007). $\log L / L_{\odot}$ of HD 73666 is taken from Fossati et al. (2008). All other data are taken from van Leeuwen (2007)

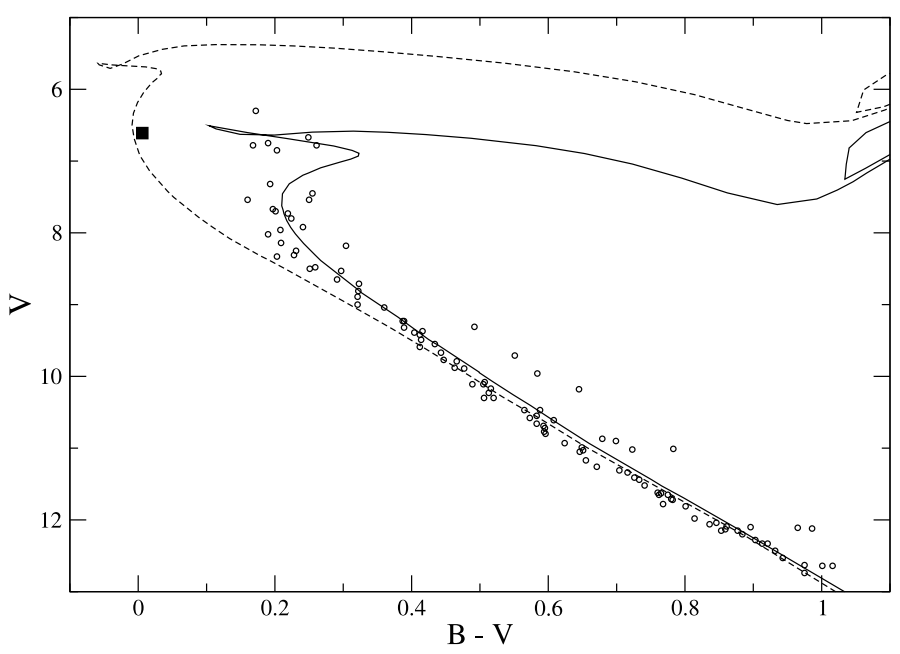

Fig. 3. Color-magnitude diagram of the Praesepe cluster. The full square indicates the position of HD 73666. The photometry was taken from Johnson (1952). The full line shows an isochrone from Girardi et al. (2002) for the age and metallicity given in the literature by González-García et al. (2006) and Chen et al. (2003) respectively $(\log t=8.85 \mathrm{dex} ; Z=0.024)$. The dashed line represents an isochrone of the same metallicity, but an age of $\log t=8.55$, that most accurately fits the position of HD 73666 in the HR diagram.

and the metallicity of the Praesepe cluster is $Z=$ $0.024 \pm 0.002$ dex (Chen et al. 2003) showing that Praesepe is not a low-metallicity open cluster. However, a few of these stars might remain unrecognised, and so we also examine this possibility.

What distinguishes horizontal branch from main-sequence stars is their mass-radius relation leading to $\log g$ values different from those typical of main-sequence stars. For horizontal branch stars at $T_{\text {eff }} \sim 9000 \mathrm{~K}, \log g$ ranges between 3.1 and 3.5 (Möhler 2004). For a star of the same values of $T_{\text {eff }}$ and $\log L / L_{\odot}$ (and hence $R / R_{\odot}$ ) that we observe, but a mass of $0.6 M_{\odot}, \log g$ would be $3.35 \pm 0.05$. Figure 4 shows the observed $\mathrm{H} \alpha$ line profile of HD 73666 in comparison with two synthetic profiles, calculated with Synth3 (Kochukhov 2007), assuming that $T_{\text {eff }}$ $=9380 \mathrm{~K}, \log g=3.78$ (dashed line), and $\log g=3.5$ (dotted line). We calculated the probability that the observed $\mathrm{H} \alpha$ line profile is fitted by the synthetic profile calculated with $\log g=$ 3.78 or $\log g=3.5$. For the higher value of $\log g$, we obtained a probability of $99.95 \%$, while for the lower $\log g$ the probability is $0.05 \%$. The lower $\log g$ value is too low, indicating that HD 73666 is very probably not a horizontal branch star appearing above the turn-off point. This statement disagrees with the conclusion of Conti et al. (1974), although their $\log g$ determination $(\log g=3.7 \pm 0.1 \mathrm{dex})$ agrees quite well with the adopted one of Fossati et al. (2007). The conclusion of Conti et al. (1974) that HD 73666 is most likely an horizontal branch star is based upon considerations of stellar evolution and more precisely that

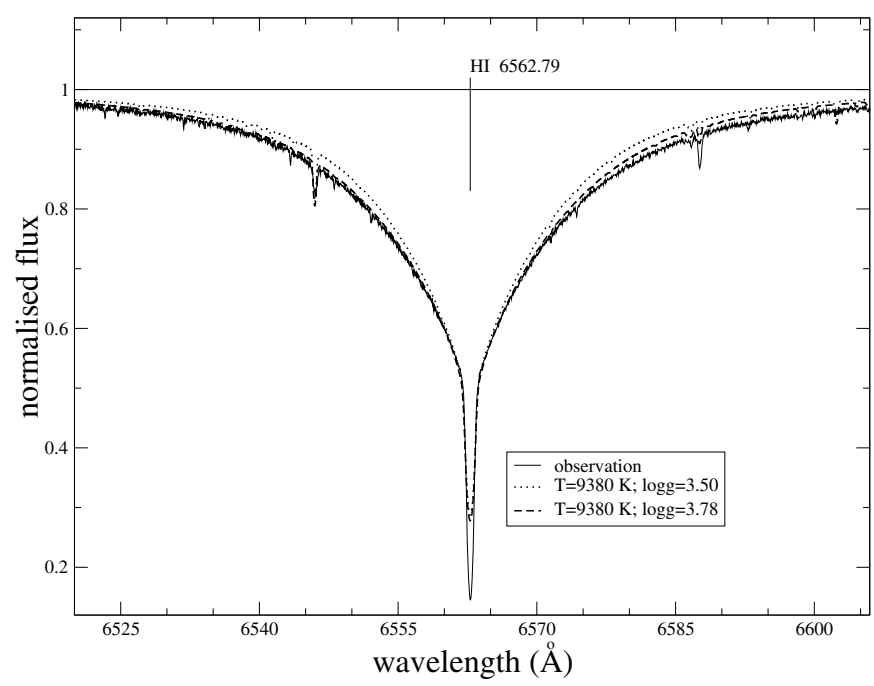

Fig. 4. Comparison between the observed and synthetic $\mathrm{H} \alpha$ line profile assuming a fixed $T_{\text {eff }}=9380 \mathrm{~K}$ and two different $\log g: 3.78$ (assumed - dashed line) and 3.5 (maximum for an horizontal branch star - dotted line).

Praesepe could statistically host one horizontal branch star. We cannot completely exclude the possibility that HD 73666 is a horizontal branch star, but we conclude that this is not very probable.

\section{Abundances}

The abundance pattern of HD 73666 was derived by Andrievsky (1998), Burkhart \& Coupry (1998), and Fossati et al. (2007). These three abundance patterns are comparable; the most complete set of determinations was given by Fossati et al. (2007) and is reproduced in Fig. 5, together with the mean abundance pattern of the A- and F-type stars member of the cluster ${ }^{1}$.

The main characteristics of the abundance pattern of HD 73666 are: a solar He abundance; an overabundance of about 0.4 dex for $\mathrm{C}, \mathrm{N}$, and $\mathrm{O}$; a solar abundance for $\mathrm{Ca}$ and $\mathrm{Sc}$, which excludes an Am classification; and a mean overabundance of about 0.4 dex for the other analysed elements ( $\mathrm{Na}, \mathrm{Mg}, \mathrm{Al}, \mathrm{Si}$, $\mathrm{S}, \mathrm{Ar}, \mathrm{K}, \mathrm{Ti}, \mathrm{V}, \mathrm{Cr}, \mathrm{Mn}, \mathrm{Fe}, \mathrm{Ni}, \mathrm{Y}$, and $\mathrm{Ba}$ ). The $\mathrm{Li}$ abundance shown in Fig. $5(+2.8$ dex, relative to the Sun) is an upper limit since at the $T_{\text {eff }}$ of HD 73666, the strongest Li line visible in the optical region (at $\lambda \sim 6707 \AA$ ) appears in a synthetic spectrum with a Li overabundance of $+2.8 \mathrm{dex}$ (relative to the Sun), and there is no trace of this line in the observed spectrum. The $\mathrm{Li}$ overabundance obtained for six Am stars around the turn-off point is about 2.1 dex (Burkhart \& Coupry 1998), too low to be detected in HD 73666, in any spectral region. Thus we do not

1 The abundances are presented as $\log \left(N_{X} / N_{\text {tot }}\right)-\log \left(N_{X} / N_{\text {tot }}\right)_{\text {solar }}$. 


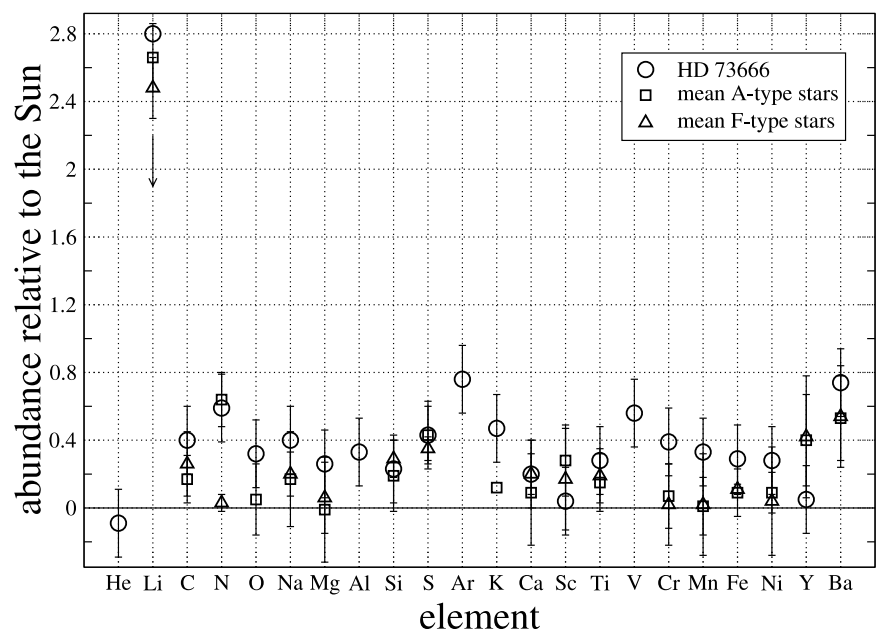

Fig. 5. Comparison between the abundances of the analysed elements obtained for HD 73666 (open circles) and the mean abundances obtained for the A- (open squares) and F-type (open triangles) stars member of the cluster. The errorbar for the abundances of HD 73666 is fixed to be 0.2 dex for all elements. The uncertainty given to the mean abundances are the standard deviations from the mean abundance. The arrow indicates that the Li abundance given for HD 73666 is an upper limit. All the given abundances are relative to the Sun (Asplund et al. 2005) and were taken from Fossati et al. (2007, 2008).

know whether the $\mathrm{Li}$ abundance is lower, similar to, or higher than that of the turn-off stars.

By applying the least square deconvolution (LSD) technique (Donati et al. 1997; Wade et al. 2000) to Stokes $V$ spectra, Fossati et al. (2007) also searched for the presence of a magnetic field. The measured longitudinal magnetic field was approximately $6 \pm 5 \mathrm{G}$, showing clearly that the star is not magnetic.

\section{Other formation scenarios}

\subsection{HD 73666 as a second generation star}

One possible formation scenario for a blue straggler is that the object is a second or even third generation star. Multiple episodes of star formation manifest themselves as separate evolutionary sequences in a cluster color-magnitude diagram. Multiple stellar populations have already been discovered for Galactic and Magellanic Cloud clusters (Piotto 2008).

The color-magnitude diagram of the Praesepe cluster, displayed in Fig. 3, clearly shows the presence of one evolutionary sequence. If a second evolutionary sequence is present, the additional episode of star formation happened at a time within the uncertainty given for the cluster age. The possibility that HD 73666 is a second or third generation star would imply the existence of a star formation mechanism that is capable of forming a single star of about $2.5 \mathrm{M} / \mathrm{M}_{\odot}$ close to the center of the cluster. We find this possibility extremely unlikely.

\subsection{Mass transfer}

\subsubsection{Distant companion}

Hartkopf \& McAlister (1984) observed HD 73666 using speckle interferometry to determine binarity, because of the overluminosity of the star, but did not detect a companion. McAlister et al. (1987) performed speckle interferometry observations of HD 73666 in 1983. They discovered the presence of a companion star at a separation $\rho=0.425 \pm 0.009$ and a position angle $\theta=127.6 \pm 0.5$ degrees at 1983.0477. Mason et al. (1993) observed the star an additional 11 times, detecting the companion only twice, on $1986.8922(\rho=0.434 \pm 0.009$, $\theta=133.7 \pm 0.5$ degrees $)$ and $1987.2664 \quad(\rho=0 " .425 \pm 0.009$, $\theta=134.1 \pm 0.5$ degrees), concluding that the star "shows little orbital motion". These measurements suggest that the companion star probably has common proper motion with HD 73666.

The secondary is $2.5 \pm 0.5$ mag fainter (Mason, private communication) than the primary star. This would imply that the secondary is an F star of about $1.5 M_{\odot}$. At a distance of $180 \mathrm{pc}$, the angular separation would mean a minimum separation at this time of about $80 \mathrm{AU}$, which suggests a period of order $450 \mathrm{yr}$ or more. The derived separation of 80 AU excludes any interaction between the two stars, unless the mutual orbit is extremely eccentric, a characteristic that would hardly survive a period of heavy mass exchange.

Binarity would have several implications. Firstly, it would favor the present location of the system at the cluster center, since Praesepe clearly exhibits mass segregation effects (Kraus \& Hillenbrand 2007). Secondly, collisions involving binary systems are an effective way of forming blue stragglers (Leonard \& Linnell 1992).

\subsubsection{Close companion}

The detection of a white dwarf or subdwarf companion close to HD 73666 would be very important, because it would clearly show that the blue straggler has been formed by mass transfer. This companion would be detectable by means of its variable radial velocity or be visible in the spectrum of HD 73666.

The presence of a small close companion (white dwarf or subdwarf) can be tested by searching for radial velocity variability. In their Figs. 13 and 14, Fossati et al. (2008) show that the star had no radial velocity variations between January 2006 and March 2007. The two measured radial velocities are also in agreement (see also Table 1) with the cluster mean. Fossati et al. (2008) also collected the radial velocity measurements obtained in the past by Abt (1970), Conti et al. (1974), Abt \& Willmarth (1999), and Madsen et al. (2002), concluding that all of these measurements are consistent with both each another and the cluster mean. In particular, Conti et al. (1974) measured the radial velocity 33 times over 14 years. They tried to fit a periodic function to their data and concluded that no periodic variation was present in the data. From these data, they derived the probability that a close companion could go undetected, assuming a random orientation of the orbital plane (see their Fig. 1). They assumed a 5:1 mass ratio (compatible with the possibility of a subdwarf or white dwarf companion) and a conservative velocity amplitude of $K_{1} \leq 1 \mathrm{~km} \mathrm{~s}^{-1}$. We note that a companion as distant as $15 \mathrm{AU}$, about the limit possible for orbital stability (Bailyn 1987) and well beyond the limit for significant mass transfer, would have an orbital velocity of about $12 \mathrm{~km} \mathrm{~s}^{-1}$. Conti et al. (1974) concluded that "for systems likely to be involved in mass exchange, the chance of their being undiscovered is remote indeed".

We extended the analysis of Conti et al. (1974) by performing a frequency analysis of all radial velocity data found in literature (65 data points), which now span a time base of about 52 years. We excluded a priori all the frequency peaks corresponding to periods shorter than 1 year, since they should have already been previously detected. Three peaks at periods of between 20 and 70 years are found, but all have a probability density (which ranges between 0 and 1) of less than 0.00002 , 
making the presence of a close companion very unlikely. We note that Fossati et al. (2007) listed HD 73666 as a singleline spectroscopic binary (SB1), an incorrect classification that should be ignored.

The presence of a hot close companion can also be checked using UV spectra. Figure 6 shows a comparison of IUE spectrophotometry of HD 73666, calculated fluxes for this star assuming that it is a single object, theoretical fluxes for a typical white dwarf ( $T_{\text {eff }}=15000 \mathrm{~K}-$ upper panel; $T_{\text {eff }}=20000 \mathrm{~K}-$ lower panel), and the sum of the two theoretical fluxes. For the white dwarf, we assumed that $\log g=8.0, R / R_{\odot}=0.013$, and the abundances obtained by Kawka et al. (2008) for BPM 6502, which has fundamental parameters similar to those assumed here. The fluxes were calculated with the use of the LTE code LLMODELS, which uses direct sampling of the line opacities (Shulyak et al. 2004) and makes it possible to compute model atmospheres with an individualised abundance pattern. Taking into account the radii of the two objects, we have derived the total flux that would be visible if HD 73666 were to have this particular white dwarf companion. The plot shows that UV fluxes would not clearly identify a white dwarf companion of $T_{\text {eff }}=$ $15000 \mathrm{~K}$, but would allow us to recognise the presence of a hotter white dwarf.

According to the cooling sequences published by Prada Moroni \& Straniero (2002), a white dwarf that formed more than half a cluster age ago, would have a luminosity of $\log L / L_{\odot} \leq$ -2 and a temperature of $T_{\text {eff }} \leq 15000 \mathrm{~K}$. Thus, the observed fluxes of HD 73666 do not exclude a white dwarf companion being present if mass transfer occurred during the first half of the cluster age, within the first $350 \mathrm{Myr}$. Figure 6 however excludes mass transfer onto HD 73666 having occurred in roughly the past 200 Myr.

On the basis of this analysis of the collected radial velocity measurements and the UV analysis, we conclude that the primary component of HD 73666 is therefore itself very likely to be a single star with only a visual companion making it a binary system. If this is not the case and mass transfer produced the blue straggler, it must have occurred at least approximately $200 \mathrm{Myr}$ ago.

\subsection{Merging and collisional formation scenario}

As mentioned in Sect. 1, Ahumada \& Lapasset (2007) reviewed the different theories of blue straggler formation. From these theories, we have already concluded that horizontal-branch confusion, multiple episodes of star formation, and mass transfer from a close companion are all quite improbable. Among the seven scenarios, the remaining possible channels of blue straggler formation for HD 73666 are collisional mergers of two stars, the merger of the two components of a close binary system, and collisional mergers between binary systems.

To consider stellar mergers and collisions as an effective way to form blue stragglers, it is important to estimate the merger and collision probability. Portegies Zwart et al. (2004) modeled the evolution of Praesepe-like open clusters using simulations that include stellar dynamics and effects of stellar evolution. Portegies Zwart et al. (2004) considered that all mergers are related to binaries: either binary coalescence due to either unstable mass transfer or perturbation by a third body. They concluded that for Praesepe-like open clusters the collision rate is about one every $100 \mathrm{Myr}$ and the merger rate of two components of a close binary system is about one every $50 \mathrm{Myr}$. Assuming an age for the Praesepe cluster of about $700 \mathrm{Myr}$, that Praesepe shows mass segregation (Kraus \& Hillenbrand 2007) and HD 73666 is
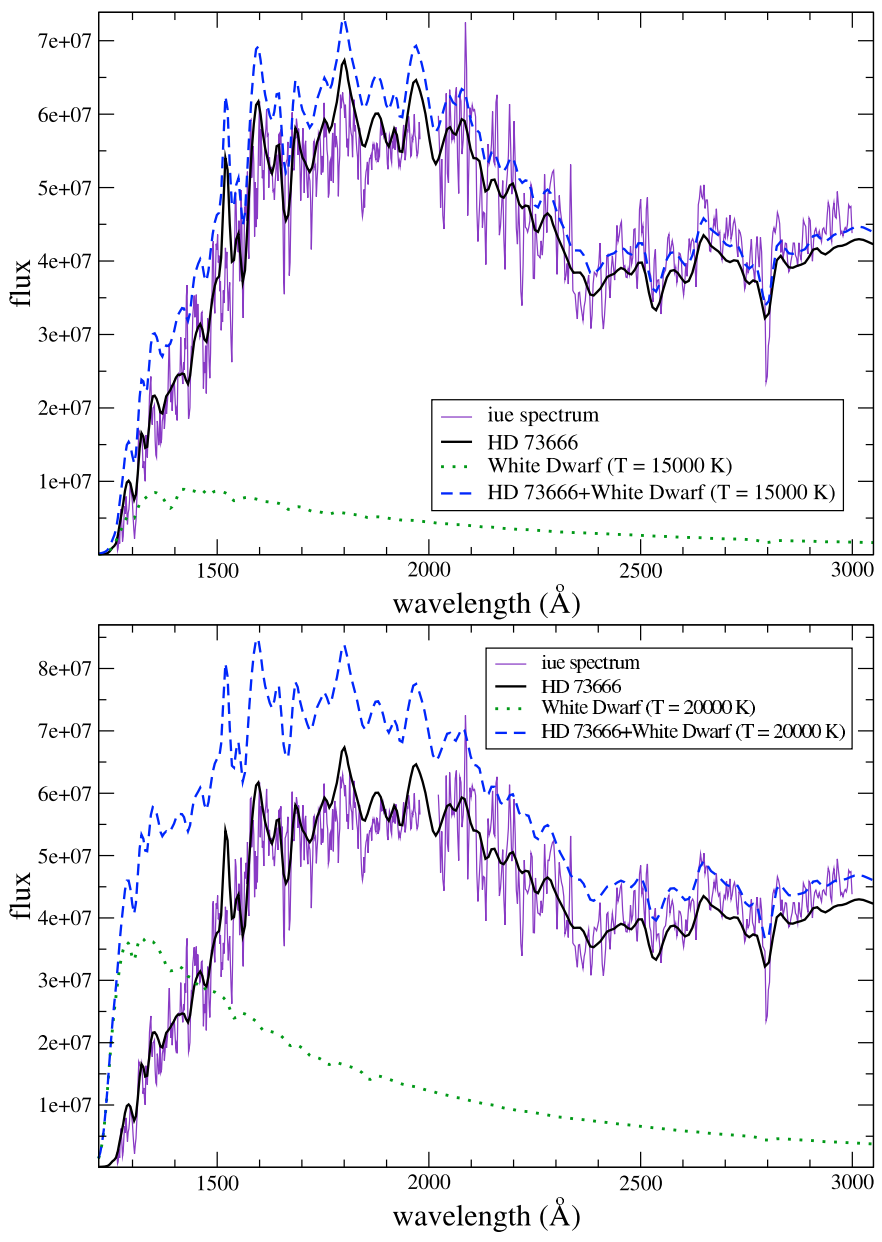

Fig. 6. Comparison between IUE spectrophotometry (thin full line) and theoretical LLMODELS fluxes for HD 73666 (thick full line), for a typical white dwarf $\left(T_{\text {eff }}=15000 \mathrm{~K}\right.$ - upper panel $/ T_{\text {eff }}=20000 \mathrm{~K}$ - lower panel, $\log g=8.0, R / R_{\odot}=0.013$, dotted line) and for the two components together (dashed line). All the theoretical fluxes take into account the estimated stellar radii. The theoretical fluxes have a different resolution $(\sim 100)$ than the IUE spectra $(\sim 900)$ for visualisation reasons.

located close to the cluster center, it is highly probable that stellar collisions and mergers have occurred within the cluster age.

Shetrone \& Sandquist (2000) derived the abundances of blue stragglers and turn-off stars in M 67, to measure chemical properties that allow us to distinguish between stragglers formed by collision or binary mass transfer. They mention that a severe lack of lithium could be an important signature of a stellar collision (Lombardi et al. 2002), although a lack of lithium is also expected in blue stragglers formed for mass transfer, so that the lithium depletion alone is not enough to indicate a collisional origin. In HD 73666, Li is also not observed and it is impossible to determine whether this is a temperature effect or represents a true lack of lithium. Shetrone \& Sandquist (2000) also concluded that $\mathrm{C}, \mathrm{N}$, and $\mathrm{O}$ may be more useful. In particular, a blue straggler formed by collision will not change the original CNO abundances, which should be similar to those of the turn-off stars. In the case of formation by binary mass transfer, $\mathrm{CNO}$ should be modified and the secondary would become a helium or $\mathrm{CO}$ white dwarf. Following the model published by Chen \& Han (2004), it is possible to deduce how much the CNO abundances of the primary star would change due to mass transfer. According to their models, the oxygen abundance should not vary, while the carbon abundance should decrease by about $50 \%$ 
and the nitrogen abundance increase by about $150 \%$. Both the nitrogen and carbon abundances of HD 73666 are comparable to those of the other A-type stars in the cluster (see Fig. 5). This result is consistent with our conclusion given in Sect. 5.2 that it is most likely that HD 73666 does not have a close companion and therefore did not undergo mass transfer. Since we have eliminated the alternatives, we conclude that HD 73666 was probably formed by either merging or a collision.

\section{Discussion and conclusion}

We now consider whether the surface CNO abundances could help us to ascertain whether a stellar collision occurred. We consider the predictions of Sills et al. (2005) for the surface helium and CNO abundance of stragglers formed after the collision of two low-mass stars $\left(0.6 M / M_{\odot}\right)$. They found that the $\mathrm{He}$ and $\mathrm{CNO}$ abundances change from the original abundances of the two colliding stars but not fast enough to be visible within $350 \mathrm{Myr}$, which is the estimated maximum age of HD 73666 since becoming a blue straggler (see Sect. 6.1).

If instead we consider a collision between a $2 M_{\odot}$ star (operating with a $\mathrm{CNO}$ cycle) and a $0.5 M_{\odot}$ star, we would expect the $\mathrm{CNO}$ present in the core to remain inside the core of the remnant star, as observed for He in low-mass stars. We note however that the probability of a collision of a high mass and a low-mass star, in forming HD 73666, is lower than the collision of two stars of masses near $1 M_{\odot}$, considering that in the center of the cluster the mean stellar mass is about $0.8 M_{\odot}$ (Adams et al. 2002). We also note that the models proposed by Lombardi et al. (1996) and Lombardi et al. (2002) exhibit a small mass loss during the collision of between 1 and $10 \%$ of the total mass of the colliding stars.

We conclude that the abundances of CNO in HD 73666, which are similar to those of the turn-off stars, are consistent with collisional formation.

Since we did not find any evidence to contradict the merging and collisional formation scenario, it is probable that HD 73666 was formed by the collisional mergers of two stars, a merger of the two components of a close binary system, or collisional mergers between binary systems. Knigge et al. (2009) showed that the most likely formation scenario in globular clusters is the merging, according to the current blue straggler statistics in globular clusters and model formation.

We emphasize that we are unable to establish which of these two formation mechanisms is the most probable for HD 73666.

\subsection{Minimum and maximum times since formation}

A minimum age can be deduced if the blue straggler was formed by a physical stellar collision. Sills et al. (1997, 2001, 2002) showed that the remnants of physical stellar collisions, in particular of off-axis collisions, should be very rapidly rotating objects with typical rotational velocities similar to or greater than the break-up velocity. This brought us to the conclusion that if blue stragglers are also formed by stellar collisions a mechanism to reduce the angular momentum must exist. Sills et al. (2005) showed that this spin-down mechanism can be effective in terms of mass loss and that the star loses about $80 \%$ of its angular momentum within $5 \mathrm{Myr}$, while a blue straggler such as HD 73666 needs about $1.4 \mathrm{Myr}$ to reach the main sequence after the collision. HD 73666 has an unusually slow rotation for an A1V star, so that if the star is actually slowly rotating the minimum age of the star as a blue straggler could be close to $5 \mathrm{Myr}$.
Fossati et al. (2007) derived a non-zero microturbulence velocity of $v_{\text {mic }}=1.9 \pm 0.2 \mathrm{~km} \mathrm{~s}^{-1}$, which is a fairly normal value for a star of this mass and appears to be indicating the disappearance of large-scale convection zones, near the surface of the star. This agrees with several model predictions of collisionally formed blue stragglers (e.g, Sills et al. 1997; Glebbeek \& Pols 2008).

The maximum age of HD 73666 since becoming a blue straggler is given by the age of the isochrone on which it lies in Fig. 3, which is about $350 \mathrm{Myr}$. If HD 73666 is an intrinsically slow rotator $\left(v \sin i \leq 90 \mathrm{~km} \mathrm{~s}^{-1}\right.$; Charbonneau \& Michaud 1991), an additional maximum age limit is provided by the time it takes for a slowly-rotating A1V star to develop chemical abundance anomalies, such as Am characteristics, by diffusion since the end of convection in its outer envelope. Talon et al. (2006) computed evolutionary models with diffusion for stars of 1.7 to $2.5 M_{\odot}$. Their most relevant model, labeled $2.5 \mathrm{P} 2$, for a star of mass $2.5 M_{\odot}$ and rotation speed $15 \mathrm{~km} \mathrm{~s}^{-1}$, shows clear abundance anomalies before $50 \mathrm{Myr}$, which we can conservatively set to represent the maximum age of HD 73666. This is, of course, valid if HD 73666 is now an intrinsically slow rotator. The fact that HD 73666 appears so far off the ZAMS does not contradict the given maximum age of $50 \mathrm{Myr}$, since collisionally formed blue stragglers do appear on the main sequence and not on the ZAMS, but at an already evolved stage.

\subsection{Conclusions}

We conclude that the Praesepe blue straggler, HD 73666, was probably formed by the physical stellar collision and merger of two low-mass stars, between 5 and $350 \mathrm{Myr}$ ago (50 Myr if the star is an intrinsic slow rotator) if current models are correct.

On the basis of our knowledge of HD 73666, it is impossible to identify either a direct stellar collision and binary coalescence as being the formation mechanism for HD73666.

HD 73666 could represent a perfect object for testing current models of collisionally formed blue stragglers. The wide and detailed knowledge available about this star and the environment in which this star is present would allow us to test the reliability of current models and to provide important constraints their future development.

Acknowledgements. L.F. has received support from the Austrian Science Foundation (FWF project P17890-N2). We thank Dr B. Mason for his help in obtaining the interferometric measurements, M. Gruberbauer for the frequency analysis and P. Reegen for the useful discussions. We are grateful to the anonymous referee for the comments and suggestions that improved a lot the manuscript.

\section{References}

Abt, H. A. 1970, ApJS, 19, 387

Abt, H. A., \& Willmarth, D. W. 1999, ApJ, 521, 682

Adams, J. D., Stauffer, J. R., Skrutskie, M. F. et al. 2002, AJ, 124, 1570

Ahumada, J., \& Lapasset, E. 1995, A\&AS, 109, 375

Ahumada, J. A., \& Lapasset, E. 2007, A\&A, 463, 789

Andrievsky, S. M. 1998, A\&A, 334, 139

Asplund, M., Grevesse, N., \& Sauval, A. J. 2005, ASP Conf. Ser., 336, 25 Bailyn, C. D. 1987, PhD Thesis, Low mass X-ray binaries in globular clusters and hierarchical triple systems, Harvard University, Cambridge, MA

Bailyn, C. D., \& Pinsonneault, M. H. 1995, ApJ, 439, 705

Baumgardt, H., Dettbarn, C., \& Wielen, R. 2000, A\&AS, 146, 251

Benz, W., \& Hills, J. G. 1987, ApJ, 323, 614

Van den Bergh, 2006, AJ, 131, 1559

Burkhart, C., \& Coupry, M. F. 1998, A\&A, 338, 1073

Charbonneau, P., \& Michaud, G. 1991, ApJ, 370, 693

Chen, L., Hou, J. L., \& Wang, J. J. 2003, AJ, 125, 1397

Chen, X., \& Han, Z. 2004, MNRAS, 355, 1182

Clampitt, L., \& Burstein, D. 1997, AJ, 114, 699 
Conti, P. S., Hensberge, G., van den Heuvel, E. P. J., \& Stickland, D. J. 1974, A\&A, 34, 393

de Marchi, F., de Angeli, F., Piotto, G., Carraro, G., \& Davies, M. B. 2006, A\&A, 459,489

Dias, W. S., Assafin, M., Flório, V., Alessi, B. S., \& Líbero, V. 2006, A\&A, 446, 949

Donati, J.-F., Semel, M., Carter, B. D., Rees, D. E., \& Collier Cameron, A. 1997, MNRAS, 291, 658

Fossati, L., Bagnulo, S., Monier, R., et al. 2007, A\&A, 476, 911

Fossati, L., Bagnulo, S., Landstreet, J., et al. 2008, A\&A, 483, 891

Girardi, L., Bertelli, G., Bressan, A., et al. 2002, A\&A, 391, 195

Glebbeek, E., \& Pols, O. R. 2008, A\&A, 488, 1017

González-Garcá, B. M., Zapatero Osorio, M. R., Béjar, V. J. S., et al. 2006, A\&A, 460, 799

Hartkopf, W. I., \& McAlister, H. A. 1984, PASP, 96, 105

Johnson, H. L. 1952, ApJ, 116, 640

Kawka, A., Vennes, S., Dupuis, J., Chayer, P., \& Lanz, T. 2008, ApJ, 675, 1518

Kharchenko, N. V., Piskunov, A. E., Röser, S., Schilbach, E., \& Scholz, R.-D. 2004, AJ, 325, 740

Kharchenko, N. V., Piskunov, A. E., Röser, S., Schilbach, E., \& Scholz, R.-D. 2005, A\&A, 438, 1163

Knigge, C., Leigh, N., \& Sills, A. 2009, Nature, 457, 288

Kochukhov, O. 2007, Spectrum synthesis for magnetic, chemically stratified stellar atmospheres, Physics of Magnetic Stars, 109

Kraus, A. L., \& Hillenbrand, L. A. 2007, AJ, 134, 2340

Kurucz, R. L. 1979, ApJS, 40, 1

Van Leeuwen, F. 2007, Hipparcos, the New Reduction of the Raw Data. Institute of Astronomy, Cambridge University, Cambridge, (UK Series: Astrophysics and Space Science Library, Dordrecht: Springer), 35020

Leonard, P. J. T. 1989, AJ, 98, 217

Leonard, P. J. T., \& Linnell, A. P., 1992, AJ, 103, 1928
Lombardi, Jr., J. C., Rasio, F. A., \& Shapiro, S. L. 1995, ApJ, 445, 117

Lombardi, Jr., J. C., Rasio, F. A., \& Shapiro, S. L. 1996, ApJ, 468, 797

Lombardi, Jr., J. C., Warren, J. S., Rasio, F. A., Sills, A., \& Warren, A. R. 2002, ApJ, 568, 939

Madsen, S., Dravins, D., \& Lindegren, L. 2002, A\&A, 381, 446

Mason, B. D., Hartkopf, W. I., McAlister, H. A., \& Sowell, J. R. 1993, AJ, 106, 637

McAlister, H. A., Hartkopf, W. I., Hutter, D. J., \& Franz, O. G. 1987, AJ, 93, 688 Mermilliod, J.-C., \& Mayor, M. 1999, A\&A, 352, 479

Mermilliod, J.-C., \& Paunzen, E. 2003, A\&A, 410, 511

Möhler, S. 2004, IAU Symp. 224, ed. Zverko, J., Ziznovsky, J., Adelman, S. J., \& Weiss, W. W., 395

Piotto, G. 2008, IAU Symp., 246, 141

Portegies Zwart, S. F., Hut, P., McMillan, S. L. W., \& Makino, J. 2004, MNRAS, 351,473

Prada Moroni, P. G., \& Straniero, O. 2002, ApJ, 581, 585

Robichon, N., Arenou, F., Mermilliod, J.-C., \& Turon, C. 1999, A\&A, 345, 471

Sandage, A., \& Wallerstein, G. 1960, ApJ, 131, 598

Sandquist, E. L., Bolte, M., \& Hernquist, L. 1997, ApJ, 477, 335

Shetrone, M. D., \& Sandquist, E. L. 2000, AJ, 120, 1913

Shulyak, D., Tsymbal, V., Ryabchikova, T., Stütz, Ch., \& Weiss, W. W. 2004, A\&A, 428, 993

Sills, A., Lombardi, Jr., J. C., Bailyn, C. D., et al. 1997, ApJ, 487, 290

Sills, A., Faber, J. A., Lombardi, Jr., J. C., Rasio, F. A., \& Warren, A. R. 2001, ApJ, 548, 323

Sills, A., Adams, T., Davies, M. B., \& Bate, M. R. 2002, MNRAS, 332, 49

Sills, A., Adams, T., \& Davies, M. B. 2005, MNRAS, 358, 716

Talon, S., Richard, O., \& Michaud, G. 2006, ApJ, 645, 634

Wade, G. A., Donati, J.-F., Landstreet, J. D., \& Shorlin, S. L. S. 2000, MNRAS, 313,823

Wang, J. J., Chen, L., Zhao, J. H., \& Jiang, P. F. 1995, A\&AS, 113, 419 